\title{
The diverse pulsational behaviour of $\beta$ Cep stars: results from long-term monitoring
}

\author{
G. Handler \\ Nicolaus Copernicus Astronomical Center, Bartycka 18, 00-716 Warsaw, Poland \\ email: gerald@camk.edu.pl
}

\begin{abstract}
We studied seven $\beta$ Cep stars photometrically over the past ten years. Some showed amplitude variations, some frequency changes, and others exhibited stable pulsations, with no consistent picture yet emerging. Additionally, 12 Lac appears to have a 6.7-yr binary companion.
\end{abstract}

Keywords. stars: early-type, stars: evolution, stars: individual (12 Lac, $\nu$ Eri, $\sigma$ Sco, $\gamma$ Peg), stars: oscillations (including pulsations), stars: rotation, binaries: general

\section{Observations and results}

This study is mostly based on measurements with the T6 automated telescope at Fairborn Observatory, Arizona (Strassmeier et al. 1997). Whereas the original idea was to collect data for asteroseismology, some interesting additional behaviour was observed...

\section{1. $12 L a c$}

For this star, we have 2381 h of data available, collected over nine seasons during 724 nights. It was noticed that the noise level in these measurements (as determined from the comparison stars) was higher than expected, especially around the known pulsation frequencies. Part of the explanation is likely binarity (Fig. 1).

However, even after correcting for the orbital light-time effect, the Fourier noise level in the data was still much higher than the measurement precision would suggest. We therefore observed $12 \mathrm{Lac}$ with the MOST satellite (Walker et al. 2003), resulting in the detection of several more pulsation modes. It turned out that the high noise level in the ground-based data can readily be explained by the presence of these new pulsation modes, with seasonally variable amplitudes. In some subsets of data these amplitudes may be high enough to even facilitate photometric mode identification.

\section{2. $\nu$ Eri}

We have collected all literature data for the star and obtained $379 \mathrm{~h}$ of new observations during 91 nights last season. The amplitude spectrum of $\nu$ Eri is dominated by the fundamental radial mode and a rotationally split $l=1$ triplet. The star also shows mild amplitude variability, but more striking are its frequency variations (Fig. 2).

\subsection{Other stars}

The pulsation spectrum of the hybrid $\beta$ Cep/SPB star $\gamma$ Peg (1497 h of data on 388 nights over six consecutive seasons) proved to be extremely stable over time, allowing to detect oscillations with amplitudes down to $0.35 \mathrm{mmag}$ in the g-mode domain and down to $0.24 \mathrm{mmag}$ at its p-mode frequencies.

$\sigma$ Sco was monitored only last season (272 h of data during 81 nights). Its pulsation amplitude has dropped to less than 1/4 of what is was in 1972 (Jerzykiewicz \& Sterken 1984). $15 \mathrm{CMa}(803 \mathrm{hr} / 250$ nights/6 seasons of new observations plus archival data) 

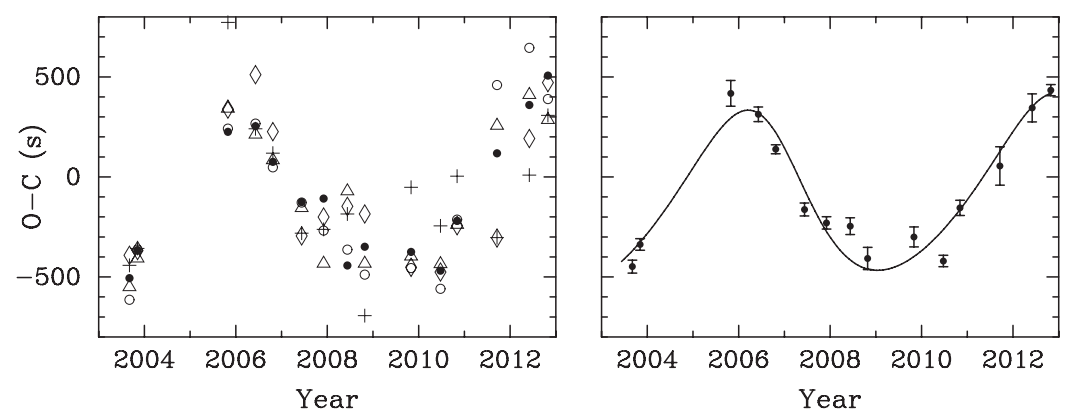

Figure 1. Left: $(\mathrm{O}-\mathrm{C})$ diagram of the four strongest pulsation modes and one combination frequency (different symbols) of 12 Lac. All vary in phase with the same amplitude. Right: orbital fit to the averaged $(\mathrm{O}-\mathrm{C})$ values, suggesting a $M>1.4 M_{\odot}$ companion in a 6.7-yr, $e \approx 0.3$ orbit.
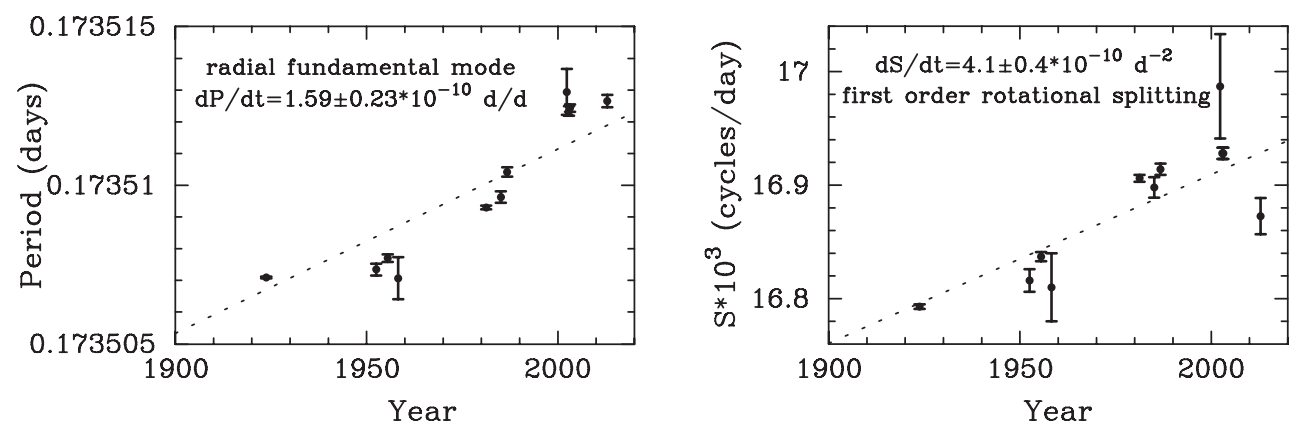

Figure 2. Left: the radial fundamental mode period of $\nu$ Eri over 90 years of measurement. The period increases, but about seven times faster than expected from stellar evolution calculations. Right: the first order rotational splitting $\left.\left(S=\left(f_{m=+1}-f_{m=-1}\right) / 2\right)\right)$ of the $l=1, g_{1}$ mode of $\nu$ Eri, indicating a temporal increase of the rotation rate.

showed a fairly stable pulsation spectrum over the last 40 years, whereas HD 167743 (590 h/217 nights/4 seasons) exhibited amplitude variations of a few, but not all, its modes. Finally, at least the strongest modes of ALS 4680 (179 h/63 nights/3 seasons, plus MOST and ASAS data) underwent considerable seasonal amplitude changes.

\section{Acknowledgements}

Part of the data discussed here were acquired by Eloy Rodríguez, Refilwe Kgoadi, and Aleksander Schwarzenberg-Czerny. Some analyses benefitted from MOST observations. This research has been supported by the Polish NCN grant 2011/01/B/ST9/05448.

\section{References}

Jerzykiewicz, M. \& Sterken, C., 1984, MNRAS, 211, 297

Strassmeier K. G., Boyd L. J., Epand D. H., \& Granzer, T. 1997, PASP, 109, 697

Walker, G., Matthews, J., Kuschnig, R., et al. 2003, PASP, 115, 1023 